\title{
Review of Surface Enhanced Raman Spectroscopic (SERS) Detection of Synthetic
}

\section{Chemical Pesticides}

3

4

5

6

Shintaro Pang ${ }^{1}$, Tianxi Yang ${ }^{1}$ and Lili $\mathrm{He}^{1 *}$

7

8

$9 \quad{ }^{1} 102$ Holdsworth Way, Department of Food Science, University of Massachusetts Amherst, MA

10 01003, USA

11

12

$13 *$ Corresponding Author

14

15

16

17

18

(C) 2016. This manuscript version is made available under the Elsevier user license

http://www.elsevier.com/open-access/userlicense/1.0/ 


\section{Abstract}

20 Pesticides are essential in modern agricultural practices. Detection of pesticides is an essential

21 step in regulating and monitoring the levels of pesticides in the environment. Even though

22 GC/LC-MS is often the gold standard method for pesticide detection, recent technological

23 advancements has promoted the creation of alternative techniques, such as Surface Enhanced

24 Raman Spectroscopy (SERS), that provide added advantages such as ultrasensitive detection,

25 faster turnover, simpler protocols, in situ sampling, on-site capability and reduced cost. In this

26 review, a comprehensive report of recent advances in SERS detection of synthetic chemical

27 pesticides is given. The development and applications of the SERS technique for pesticide

28 detection in both simple and complex matrices are discussed. The main advantages of using

29 SERS for pesticide detection are highlighted, together with its limitations. Lastly, promising

30 future trends and applications of SERS for pesticides detection are also discussed.

31

32 Keywords: SERS; pesticide; detection; substrate; method; matrix 


\section{Contents}

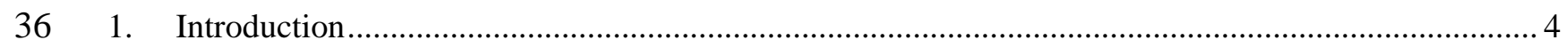

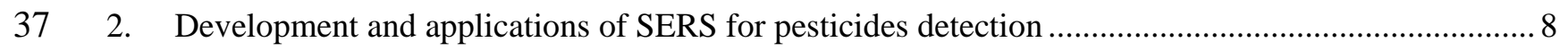

38 2.1. Initial development of SERS for detection of pesticide in simple matrices............................... 8

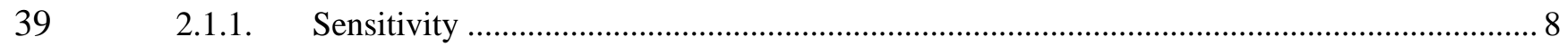

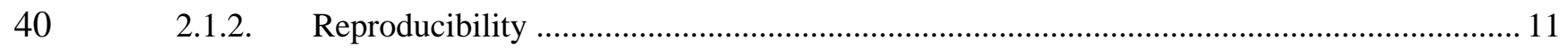

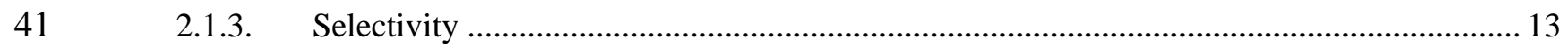

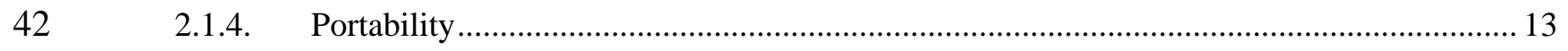

$43 \quad$ 2.2. Further applications of SERS for pesticide detection in real complex matrices ........................ 15

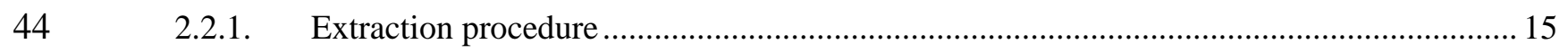

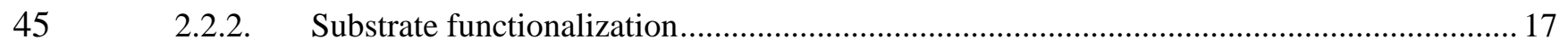

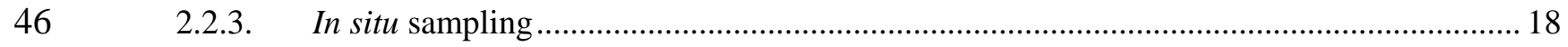

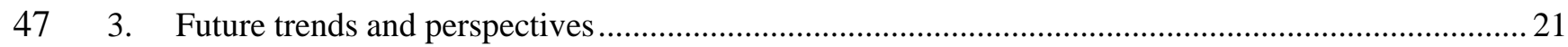

$48 \quad 3.1$ Commercialization and method standardization ................................................................... 21

$49 \quad$ 3.2. Method integration and advance of instrumentation............................................................. 24

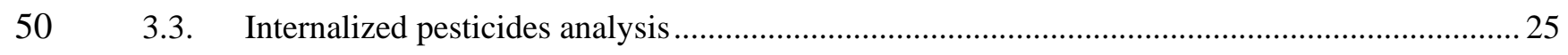

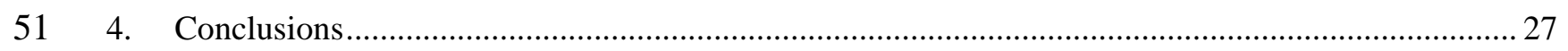

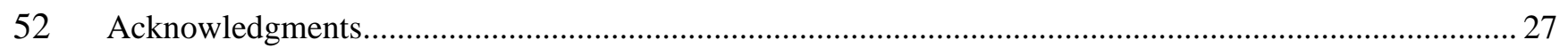

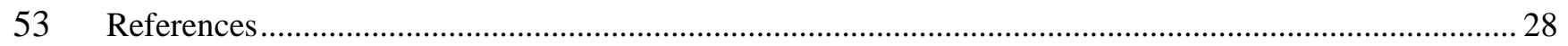

54

55

56

57 


\section{Introduction}

59 A pesticide is any substance or mixture of substances intended for preventing, destroying, 60 repelling, or mitigating any pest. Because pests and diseases damage up to one-third of crops 61 during growth, harvest or storage, pesticides are essential in modern agricultural practices. The

62 use of pesticides in commercial agriculture has led to an increase in farm productivity[1].

63 According to the Environmental Protection Agency (EPA) report, the agricultural market shares

64 of total pesticides resulted in \$35.8 billion in 2006 and more than \$39.4 billion in 2007 in

65 spending expenditure respectively [2].Based on their chemical structures and functionality,

66 synthetic pesticides are classified into five classes: organochlorine, organophosphate, carbamate,

67 neonicotinoid and pyrethroid. Biopesticides, which is another category of pesticides, is derived

68 from biological materials, and hence, their chemical compositions can be widely varied, such as

69 a single protein to a microorganism that attacks pests.

71 Most chemical pesticides are designed to be toxic to pests, so by their very nature they pose

72 risks to human beings, wildlife and the environment. Acute toxicity data of most pesticides are

73 well documented. On the other hand,chronic toxicity data of pesticides are more difficult to

74 obtain, and this has raised public health concerns nowadays. In order to monitor the amount of

75 pesticides and metabolites accumulated in nature, a wide range of sample types, including

76 forensic, crops, environmental (e.g. soil, water, air), food, beverage, biological (e.g. blood), plant

77 and animal-derived products are needed to be tested routinely.

A critical requirement for pesticide monitoring is to have a well-established detection method.

80 The detection technology of choice must possess several key characteristics, such as excellent 
81 sensitivity and reproducibility. Since the $20^{\text {th }}$ century, these important detection characteristic

82 requirements have been fulfilled by various chromatographic based techniques, including

83 LC/GC-MS, HPLCand TLC [3-6]. Even though these methods have been evolving gradually to

84 improve on their detection capabilities, other detection technologies have also emerged that

85 promise added benefits, such as faster detection times, simpler protocols, in situ sampling,

86 portability and reduced cost. Surface enhanced Raman spectroscopy, or SERS, is one of these

87 techniques, and will be the focus of this review for the detection of synthetic chemical pesticides.

89 SERS is essentially an agglomeration of two techniques, namely Raman spectroscopy and

90 nanotechnology. Raman scattering was first discovered in 1928 by Raman and Krishnan, who

91 observed the inelastic scattering of light which constitutes only about one in a million photons of

92 incident light striking a surface. The rest reflect elastically, commonly known as Rayleigh

93 scattering. It was further discovered that the frequency changes that occurred due to the inelastic

94 scattering of light matched precisely with the differences in vibrational energy levels. This

95 enables every type of molecule to yield distinct Raman spectral profiles since different functional

96 groups possess different characteristic vibrational energies. Thus, the main advantage of Raman

97 spectroscopy is the capability for molecular fingerprint specificity for every distinct

98 molecule/analyte. However, this method was not applicable for sensitive detection due to the

99 inherently weak Raman signals. In 1970, researchers discovered that Raman signals were

100 enhanced by $10^{4}-10^{5}$ if the target analyte was placed in close proximity to a roughened noble

101 metal substrate. Although the exact mechanism for this phenomenon is not clearly understood,

102 two sets of theories have been well-received by the scientific community; namely the

103 electromagnetic and chemical theory. In the electromagnetic theory, the enhancement of Raman 
104 signals is thought to be due to the excitation of the localized surface plasmon resonance (LSPR)

105 of nanoparticles when an incident light hits the surface of the target analyte close to the

106 nanomaterial. In order to maximize the enhancement of Raman signals, the excitation frequency

107 of the nanomaterials used has to resonate with that of the incident light (i.e. for noble metals, this

108 is in the UV-vis range). This will then result in intensity peaks at particular Raman shifts with at

109 least $10^{4}-10^{6}$ enhancements. In the chemical theory, the Raman signal enhancement occurs

110 based on the assumption that the target analyte is adsorbed on the metal surface and that a charge

111 transfer is in effect. Because of the chemisorption of target analyte on the substrate, the

112 electronic state of the complex is shifted to a new absorption maximum, which allows it to

113 resonate with the laser excitation frequency, and thus enhance the Raman signals. Several recent

114 review articles are available to get deeper understanding of the mechanisms and theory of 115 SERS[7-9].

117 Due to the potential of SERS as a detection technique, several scientific articles and review 118 papers have also been published to highlight its possible applications. Examples include the 119 detection of target compounds such as food chemicals [10], environmental pollutants [11], 120 biomolecules[12] and cancer diagnostic agents [13], as well as to investigate broader topics such 121 as forensic science [14],food safety [15] and integration with other technologies such as 122 microfluidics [16]. General reviews of SERS applications are also available[17,18]. Although 123 pesticides detection was covered briefly in some of these articles, a comprehensive report of 124 recent developments in SERS technique specifically for pesticides detection has not yet been 125 reported. 
128 Vo-Dinh [19]. In their article, eight organophosphorous pesticides were characterized using

129 silver (Ag)-coated microspheres as substrates. Since then, a large number of scientific

130 publications regarding the SERS detection of pesticides have been published (Figure 1). In this

131 review paper, a comprehensive report of SERS for synthetic chemical pesticide detection is

132 given. The initial development of SERS for pesticides detection mainly focused on the SERS

133 substrate development, with demonstration of its detection capability using pesticides. In these

134 studies, pesticides in simple matrices were tested. Hence, the first segment covers several aspects,

135 including the sensitivity, reproducibility, selectivity, and portability of SERS that have been

136 improved for pesticide detection mainly as a result of recent advances in substrate development.

137 Another critical step for the advancement of SERS as a detection tool is the ability to selectively

138 detect a target in any matrix. Therefore, the second segment focuses on applications of SERS for

139 pesticide detection in complex matrices. Advantages and limitations of using SERS for

140 pesticides detection are highlighted. Lastly, promising future trends and applications of SERS for

141 pesticides detection are also discussed.

142

143

144

145

146 


\section{$147 \quad 2 . \quad$ Development and applications of SERS for pesticides detection}

148 2.1. Initial development of SERS for detection of pesticide in simple matrices

One of the main advantages of using SERS over traditional analytical techniques for the

150 detection of pesticides in simple matrices (e.g. water or organic solvents) is the speed at which

151 samples can be analyzed. The protocol can be as simple and quick as dropping a microliter

152 sample onto a SERS substrate followed by a split-second laser integration time to obtain a SERS

153 signal and comparing it against a reference spectrum. Other important considerations include

154 sensitivity, reproducibility, selectivity, and portability. These factors have been improved over

155 the years and are mainly driven by the development of SERS substrates.

\section{$157 \quad$ 2.1.1. Sensitivity}

The limit of detection (LOD) is perhaps the most widely studied characteristic in a SERS

159 study forthe sensitive detection of pesticides. The reported LODs of SERS for pesticides

160 detection in simple matrices (e.g. water or organic solvents) mainly depends on the type of

161 substrates and the pesticide molecular structure. Another important thing to remember is that the

162 LODs for pesticides detection, which aregenerally reportedin the parts-per-million (ppm) or

163 parts-per-billion (ppb) level, cannot be directly compared to the maximum residue levels (MRLs)

164 allowed in agricultural produce and the environment, which are set by regulatory agencies such 165 as EPA. The MRL for a specific pesticide is based on the sample matrix it is present in, while the

166 LOD for the pesticide is usually based on the concentration of that pesticide in its solvent.

167 Therefore, careful transition of the units and discussion of the relationship between LOD of the

168 analytical method and the governmental requirement of the pesticide detection are critically

169 needed. 
171 largely be attributed to the variations in "hot spots" on substrates [20]. These hot spots are found

172 at the interstitial gaps between metallic nanoparticles,and are able to produce intense local field

173 enhancements due to localized surface plasmon resonance (LSPR).The LOD of SERS methods

174 can sometimes reach a single molecule detection level through a rational design of the SERS

175 substrate and the experiment[21-24].

Thus, efforts have been made to increase the number, strength and location of hot spots to

177 lower the LOD of pesticides. In the case of Ag or gold nanoparticles(Au NPs), i.e. most widely

178 used SERS substrate, the addition of salt, such as $\mathrm{NaCl}$, is used as an aggregating agent to

179 increase the number of hot spots. In this way, the electric charges surrounding nanoparticles are

180 neutralized by the salt and is able to pull both nanoparticles and the adsorbed target together,

181 which will subsequently enhance the SERS peak intensities [25]. Pre-concentration of

182 nanoparticles can also be done through physical means such as filtration. Yu and White showed

183 the concept by trapping Ag NPs on filter membranes in order to form a SERS active substrate,

184 after which a large volume $(\mathrm{mL})$ sample containing low levels of malathionor melamine were

185 concentrated onto the filter [26]. Another example is the coupling of electrokinetic pre-

186 concentration with SERS, which was developed to detect small molecules such as antibiotics and

187 phenols $[27,28]$. Besides increasing the number of hot spots, alternations of the shape and size of

188 the nanoparticles can also increase the strength of localized electromagnetic field (hot spots)

189 and/or increase the likelihood of the contact of target pesticides with the hot spots. Ag NPs that

190 have aggregated forms of nanosize shapes such as flowers and leaves have been fabricated to

191 take advantage of their SERS enhancement capabilities [29,30]. Unique nanoparticle shapes

192 synthesized for SERS substrate applications include nanowires, nanocube and nanodishes [31- 
193 33]. By increasing the probability of the target landing at the centroid position of a hot spot, 194 ultrasensitive detection even at a single molecule has been achieved under optimal SERS 195 conditions [21].

From the pesticide point of view, the sensitivity of SERS is different for each

198 pesticidemainly because of the unique, intrinsic vibration of the molecules, the interaction 199 between pesticide molecules and the substrate, as well as the compatibility of the pesticides 200 when complexed with the substrate. For the intrinsic vibration, molecules with conjugated 201 double bond system and symmetric vibrational modes are more active than the others. Therefore, 202 certain pesticides such as crystal violet and malachite green are more popular as pesticide targets 203 for the evaluation of a SERS substrate [34,35]. Pesticides with certain functional groups (e.g 204 thiol, amine) that can bind Au and Ag substrates strongly are also good targets. For example, 205 ferbam, thiram, thiabendazole and phosmet have been used for substrate evaluation partly for 206 this reason [36-39]. Particularly for ferbam and thiram, since there is no GC/LC-MS method 207 available to detect them directly, the capability of using SERS as the detection method show 208 great promise [40,41]. Many pesticides intrinsically possess the two properties mentioned above, 209 thus making them promising targets for sensitive SERS analysis. In the case of compatibility, if 210 the affinity of the target to substrate is too weak for adequate adsorption, the sensitivity of SERS 211 detection can also be dramatically reduced. For example, hydrophobic molecules such as

212 polycyclic aromatic hydrocarbons (PAHs) and polychlorinated biphenyls (PCBs) will not adsorb 213 well on citrate stabilized Au or Ag colloids due to the incompatibility of the surface chemistry

214 [42]. Hence, attempts have been made to overcome this limitation by modification of the 215 substrate surface. In the case of hydrophobic molecule detection, colloidal hydrophobic films 
216 have been placed onto Au NPs immobilized onto silanized quartz substrates to detect PAH [42].

217 Kubackova et al detected four organochlorine pesticides (i.e. Aldrin, dieldrin, lindane, and $\alpha-$

218 endosulfan) using a SERS substrate modified with several types of alkyl dithiols to increase the

219 affinity of these liposoluble pesticides [43]. Due to the higher selectivity of these pesticides to

220 the surface modified SERS substrate and the interparticle linkage induced by the thiol groups to

221 produce more "hot spots", the LOD was able to reach $10^{-8} \mathrm{M}$. Zheng et al showed the advantage

222 of using an oleate-modified $\mathrm{Fe}_{3} \mathrm{O}_{4} @ \mathrm{Ag}$ microspheres due to the selectivity of the oleate group to

223 detect thiram through surface ligand exchange [44]. In their study, a gel reaction system

224 containing oleate was used to avoid aggregation of $\mathrm{Fe}_{3} \mathrm{O}_{4} @ \mathrm{Ag}$ microspheres and to modify

225 synthesized Ag NPs simultaneously during the generation process, thus increasing the sensitivity

226 of the SERS substrates. Graphene, proteins and DNA fragments have also been conjugated onto

227 SERS substrates to modify the surface chemistry to enhance the sensitivity of SERS [45-47].

228 Many of these studies show proof of concepts, but are not well studied in complex matrices such

229 as food, hence, there is still a need for application based studies to evaluate SERS for practical

230 applications of pesticide detection in real complex matrices

231

232 2.1.2. Reproducibility

233 Despite the huge advantage of SERS as an ultrasensitive detection tool, one of the main

234 limitations that continue to be addressed in SERS studies is the reproducibility of results,

235 particularly with regards to peak intensities. Thus, quantitative studies remain a challenge in

236 SERS, not only in the pesticide detection area, but generally in all kinds of SERS applications.

237 The origination of the variation mainly arises from the substrates and sample preparation that

238 produce and control the hot spots. The hot spots of the traditional colloid SERS substrates 
239 highly depend on their aggregation. Despite wide commercial availability and relative low cost,

240 they usually have difficulty in controlling aggregation, thus producing larger signal variation

241 compared to the well-ordered nanostructures. Therefore, the selection of detection spots cannot

242 be completely blind. With a Raman microscope, we are able to select certain areas on the

243 substrate for minimizing the variation. Core-shell based colloid nanoparticles have significantly

244 improved the signal variation of SERS, as their hot spots are not determined by aggregation [48].

245 Other studies that have shown to control the uniformity of nanoparticles into consistent patterns

246 include incorporation of colloids into a sol-gel [49,50] and integration with a microfluidic device $247[34,51]$.

248 Information on the reproducibility of the well-ordered nanostructures made through top-

249 down and bottom-up techniques can be found in several review papers $[52,53]$ and are not

250 discussed in this review. However, they are relatively expensive to fabricate and not as widely

251 available, which has limited their application for routine analysis in food and environmental

252 samples.

253 Another reason for the limitation on reproducibility is because each researcher often uses

254 a different Raman instrument system and employs a variety of possible configurations. For

255 example, four referenced publications using gold colloids as their SERS substrates[54-57]

256 usedRaman instruments that were completely set up differently. In addition to hardware

257 differences (i.e. LabRAM ARAMIS Raman, RamanStation 400F, Renishaw RM1000 Raman,

258 DXR Raman), their laser wavelength (i.e. 633, 780, $785 \mathrm{~nm}$ ), laser power (i.e. 0.325, 5, 20, 250

$259 \mathrm{~mW}$ ), accumulation time (i.e. $2,5,10,15 \mathrm{~s})$ and spectral resolutions $\left(1,5 \mathrm{~cm}^{-1}\right)$ were varied. The

260 lack of standardization for SERS detection of pesticides makes it challenging to compare results

261 from one lab to the other. 


\subsubsection{Selectivity}

When multiple pesticides are present in a sample, the selectivity of SERS to detect each of

265 the specific pesticide in the mixture simultaneously is a big challenge, even if the matrix was just

266 water or an organic solvent. In theory, one might think that SERS should be capable of detecting

267 multiple pesticides from a sample mixture as long as each pesticide produces a distinct SERS

268 peak. Especially with advanced chemomatrices, separation and detection of multiple pesticide

269 peaks is possible. In reality, however, SERS studies have been limited to detecting less than five

270 pesticides simultaneously each time $[57,58]$. This is probably because competitive adsorption to

271 the SERS substrates occurs when multiple analytes are present. In other words, the target

272 compounds with higher binding affinity to the substrate will have greater surface coverage

273 adsorption on the substrates, thus the concentration ratios of target compounds present in the

274 sample matrix would not be proportional to the SERS peak intensities generated. In some cases,

275 the pesticides may not even produce a significant peak due to the presence of another compound

276 with several orders of magnitude higher affinity to the substrate. Pre-separation of individual

277 pesticides or pre-treatment to reduce the number of pesticides for a single SERS test can solve

278 this problem, but this would inevitably increase the analytical time and complexity [59]. More

279 discussion can be found in the next section when talking about the detection in real matrices.

\subsubsection{Portability}

One of the popular aspects of SERS development is the potential for it to become a field

284 detection method, which is a great advantage when compared to the GC/LC-MS methods. In 
285 pesticide applications, field detection is strongly needed for rapid screening and monitoring of

286 pesticides in environment, agriculture production, and industrial processing. In order to have a

287 portable detection system, it has to be rapid, light, easy-to-use and small.

290 enabled portable SERS detection to become a reality. However, just as crucial as the instrument 291 is the development of suitable substrates. For portable applications, traditional colloidal based 292 substrates may not work well due to the additional steps needed to deposit the nanoparticles on a 293 solid surface and to concentrate/aggregate the substrate. Hence, solid SERS substrates are more 294 suitable for detection that requires portability. Another approach has been the development of 295 paper-based [60-62] or fiber-based [63,64] SERS sensors. For example, one study screen printed 296 SERS active nanoparticles onto a filter paper, which could then be used to screen many samples 297 using high throughput analysis in a portable setting [62]. Not only were these substrates cost298 effective, but they were able to exhibit reproducible results with less than $10 \%$ spot-to-spot 299 variation. In another study, a template guided self-assembly of Au NPs into ordered arrays of 300 uniform clusters was prepared on an optic fiber faucet [64]. These SERS enabled optic fiber 301 showed high-performance of SERS as demonstrated by using crystal violet. This batch method 302 approach may pave a way for low-cost, efficient SERS substrates. Continued research will be 303 needed to examine the stability of the substrates and to apply it with wider varieties of target 304 analyte. 


\subsection{Further applications of SERS for pesticide detection in real complex matrices}

For any detection method development that will eventually be used in applications, the matrix in which the target is embedded in plays a crucial factor in influencing the sensitivity and

309 reproducibility of the result. For example, for the detection of food contaminants, the matrix

310 could be aqueous (e.g. milk, fruit juices, drinking water) or solids (e.g. meat, fruits, vegetable,

311 cereal). Depending on the matrix, the interference of SERS signals can differ greatly. Since

312 SERS can detect most compounds that are in close proximity to the substrate, non-targeted

313 substances may produce substantial noise peaks to lower the sensitivity of the target analyte.

314 Furthermore, several molecules may produce strong Raman peaks that are similar to the target,

315 making it challenging for qualitative and quantitative detection. A summary of recent studies

316 using SERS to detect pesticides in complex matrices are listed in Table 1. Here, we discuss

317 several strategies that have been utilized in order to reduce the influence of complex matrices in

318 the SERS method (Figure 2).

319

\subsubsection{Extraction procedure}

321 Depending on the nature of the target pesticides and the complexity of the sample matrix,

322 in some studies, little or no extraction was applied for SERS detection of pesticides in complex

323 matrices. For example, acetamiprid was detected in commercially bought apple juice spiked with

324 acetamiprid with no prior sample treatment [65]. Even though there were significant peaks

325 coming from apple juice, characteristic peaks attributed to acetamiprid were still observed. In

326 this case, a sharp apple peak was observed in $610 \mathrm{~cm}^{-1}$ while a pure acetamiprid solution

327 contained a sharp peak at $640 \mathrm{~cm}^{-1}$. As the acetamiprid concentration in apple juice increased,

328 the apple peak shifted towards $640 \mathrm{~cm}^{-1}$, suggesting more displacement of the apple juice 
component by acetamiprid on substrate surface. The LOD was 3 ppm. A similar study was also

330 conducted to detect carbaryl using a different substrate [66]. The successful detection of

331 pesticides without extraction really depends on the particular pesticides that can bind on the

332 SERS substrate stronger than most of the matrix components, as well as a sharp and

333 characteristic peak that can be easily distinguished from the matrix peaks.

For most applications in complex matrices, extraction or separation of target pesticides are necessary before applying SERS. As SERS is not a separation technique, but a detection

337 technique, the integration of a separation technique like chromatography can greatly improve the

338 selectivity of SERS. However, the integration of chromatography and SERS detection cannot

339 compete with the current UV or mass spectroscopy detection systems in terms of instrumental

340 automation and cost-effectiveness. Further development in instrumentation is needed for this

341 type of method integration. On the other hand, a more practical strategy is to adopt and modify

342 the sample clean-up procedures that are used for GC/LC-MS before applying SERS. Compared

343 to chromatographic methods such as LC/GC-MS, a great advantage of SERS is that sample

344 preparations do not require high purifications of samples, thus simpler extraction procedures can

345 be applied to reduce time and resources. This is possible because there are no capillary tubes or

346 filters to go through; hence clogging of the instrument is not an issue.

He et al demonstrated the simplicity of pesticide SERS detection in foods by using a

349 simple swab method on apple peels [65]. A surgical swab pre-soaked in methanol solvent was

350 used to swab a $1 \mathrm{~cm}^{2}$ area on apple peels spiked with thiabendazole. They were able to

351 successfully extract the pesticide and detect as low as $0.1 \mathrm{ppm}$. Kim et al also did a similar 
352 extraction procedure for the detection of thiabendazole and chlorpyrifos by rinsing the spiked

353 apple peels in water and dropping the rinse onto their substrate [38]. Similarly, Khlebstov et al

354 reported the extraction of thiram for SERS analysis by immersing the spiked apple peels in

355 methanol and dropping the extract directly on fabricated Au nanoisland films. Despite noise

356 signals coming from other components on apple peels, the pesticide signals were distinguishable

357 and sharp enough even at $30 \mathrm{ppb}$ [67]. As we can see from all these examples, SERS is able to

358 detect pesticides in lightly treated samples as long as the matrix components do not interfere with

359 the pesticide signals. Other studies have also extracted pesticides in a similar manner [66,56].

\subsubsection{Substrate functionalization}

Another approach to detect specific pesticides in a complex matrix is through

364 functionalization of the SERS substrates. The selectivity of SERS can be improved by using

365 specific target capture agents such as aptamers (i.e. "nucleic acid antibodies") and molecularly

366 imprinted polymers (i.e. MIPs, “plastic antibodies”). Pang et al described an aptamer-based

367 SERS method that was capable of detecting four organophosphate pesticides (phorate,

368 profenofos, omethoate, isocarbophos) in apple juice. By using a Ag dendritic substrate

369 conjugated with a thiol-modified ssDNA aptamer and a blocker molecule, the apple juice

370 compounds were unable to adsorb onto the substrate surface, and thus did not contribute to the

371 SERS signal [58]. In another study, an aptamer conjugated polymer-Au NP composite

372 microsphere was used as a SERS substrate to selectively capture the pesticide malathion in tap

373 water [68]. The controlled aggregation of Au NPs on the polymer microspheres also allowed for

374 more reproducible SERS signal intensities. 
376 Although functionalization of SERS substrates can improve the selectivity, additional

377 signals can be produced from the capturers which may overlap with the target signals.

378 Sometimes, due to the formation of a complex between the capturer and pesticide molecules, the 379 SERS patterns may change significantly. Therefore, careful identification of the target pesticide

380 signals is very important. In addition, due to the additional layer on the substrate, the target 381 pesticide molecules are not directly on the surface of SERS substrate. This would inevitably

382 decrease the sensitivity because the enhancement is strongly dependent on the distance between

383 the target and substrate. The typical enhancement zone is within $10 \mathrm{~nm}$ [69]. Therefore, any

384 capturer that is larger than that is not applicable to be used in the SERS substrate

385 functionalization for pesticide detection.

Although functionalization of substrates for the detection of pesticides in several matrices 388 have been explored and proven to be of potential, its detection capability is still in its infancy.

389 More research would be needed to substantially claim the advantages of functionalization and to 390 overcome possible limitations such as contribution of modifiers to spectral signatures and 391 oversaturation of substrate surface coverage.

\subsubsection{In situ sampling}

Another popular trend in pesticide detection analysis is in situ sampling. This method 396 requires little to no sample preparation since the pesticide is detected directly on the matrix itself. 397 Coupled with a portable instrument, insitu sampling and detection find its market niche for rapid 
398 screening of pesticides in agriculture applications. The various kinds of SERS substrates that

399 have been used for in situ pesticides detection are presented in Figure 3.

$400 \quad$ One simple example of this application was illustrated in a detection assay that identified

401 pesticides on fresh tea leaves and apple peels [57]. In this study, commercially available, citrate

402 stabilized Au NPs were dropped onto the surface of the plant that was spiked with various

403 amounts of organophosphates, pyrethroids, and neonicotinoids pesticides. After mixing the

404 aqueous Au NPs solution and the spiked pesticide surface using gentle pipetting,the mixture was

405 dried and a Raman spectro-microscope was used to focus directly on the surface of the leaves

406 and peels to record their Raman signals. No pesticide Raman signals were observed when Au

407 NPs were not dropped onto the sample surface whereas intense pesticide peaks were seen with

408 the introduction of Au NPs, owing to the surface enhancement effect. Furthermore, no significant

409 background interference was observed on both fresh tea leaves and apple peels, hence, low

410 detection limits (ppb levels) for all the pesticides tested were achieved. In a similar study, an in

411 situ method for detecting malathion pesticide was introduced using SERS imaging and

412 multivariate curve resolution [55]. In this study, colloidal Au NPs solution was dropped onto

413 spiked tomato peels and Damson plum surfaces, followed by addition of a $\mathrm{NaCl}$ solution. Then a

414 mapping region was selected for SERS and data analysis. Liu et al proposed a different strategy

415 for in situ detection, whereby Ag NP films were fabricated directly on contaminated sample

416 surfaces using an in situ reduction method, followed by SERS analysis of that surface [70].

417 Based on this methodology, the pesticides paraquat and fenthion were detected in situ on the

418 surfaces of capsicum, celery and cole, as well as malachite green on fish scales. 
Another approach to the in situ sampling of pesticides on fruit surfaces was illustrated 421 using a SERS derived concept termed as "SHINERS" (Shell isolated nanoparticle-enhanced

422 Raman spectroscopy) [48]. In this detection assay, fabricated nanoparticles which consist of an 423 ultrathin coating of silica or alumina (2 nm) on Au NPs were spread onto a sample surface as a 424 "smart dust". The coating prevented agglomeration of the nanoparticles and allowed monolayer 425 distribution of the nanoparticles on the sample surface. To illustrate this concept, parathion was 426 detected on an orange peel by simply spreading the "smart dust" on the surface. An advantage of 427 this method is that it protects the SERS active metal nanostructure from touching directly onto 428 the sample and allows it to conform to the structure of the sample surface. In a separate study, 429 silver coated Au NPs (Au@Ag NPs) were characterized and shown to provide strong SERS 430 signals when applied in situ onto pesticide spiked fruit peels [71]. An interesting observation was 431 that the Raman intensity enhancements were dependent on the Ag shell thickness instead of the 432 aggregation status of nanoparticles, suggesting that "hot spots" were not the main contributor in 433 this particular SERS substrate. Rather, Au@Ag NPs acted as stand-alone-particle Raman 434 amplifiers capable of detecting trace pesticide levels on complex matrix surfaces, similar to the 435 mechanism of tip enhanced Raman spectroscopy (TERS) [72].

In a different attempt to perform in situ detection that was both sensitive and highly 438 reproducible, $\mathrm{Au}$ NPs were grafted onto dendritic $\alpha-\mathrm{Fe}_{2} \mathrm{O}_{3}$ to test thiram residues (5 $\left.\mu \mathrm{M}\right)$ on tea 439 leaves [73]. The dendritic nanostructures not only enabled the researcher to locate the test spot 440 easily through an optical microscope, but provided a highly uniform substrate pattern that is a 441 precursor to reproducible Raman enhancements. 
444 evident that this method has potential for on-site screening capability and portability. However,

445 more studies will have to be conducted such as testing it with a wide range of pesticides using

446 commonly available or cost effective substrates in order for widespread applications to become a

447 reality. Furthermore, the sample surfaces that were used were relatively clean and free of other

448 interference compounds, hence it would be interesting to see the effect of this method in real

449 matrices that may have more contamination or have been deliberately contaminated with other

450 compounds.

\section{$454 \quad$ 3. Future trends and perspectives}

\subsection{Commercialization and method standardization}

Just a few years ago, there were only two commercially available SERS substrates ready

458 for use. Both of these, Klarite ${ }^{\mathrm{TM}}$ (Renishaw Diagnostics, Glasgow, UK) and Q-SERS (Nanova,

459 Columbia, MO, USA) were manufactured based on electron beam lithography, a relatively

460 expensive and time consuming method of production. As a result, the cost of each substrate was

461 extremely high $\left(<\$ 100 /\right.$ in $\left.^{2}\right)$. Although the sensitivity and reproducibility of these substrates were

462 satisfactorily good, the cost was the biggest hindrance to widespread usage such as for quality

463 assurance and food industry applications. Studies have been conducted with these substrates to

464 show the efficacy for the detection of pesticides, such as carbaryl, phosmet, and azinphos-methyl

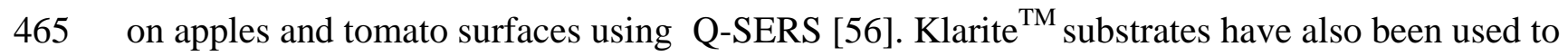


466 detect phosmet on orange skin peels [74]. Other pesticide detection studies have also been

467 conducted [75-77].

468

Due to the increasing popularity of SERS for trace detection, other commercial SERS

470 substrates have also emerged. These include Ocean Optics SERS substrate (Ocean Optics,

471 Dunedin, FL) which is basically Au NPs deposited onto a plain glass slide; SERStrate (Silmeco,

472 Copenhagen, Denmark), a silica based substrate coated with Au or Ag with an overall pili

473 nanostructure; Horiba Scientific SERS substrates (Horiba Scientific, Kyoto, Japan); P-SERS

474 (Diagnostic anSERS, College Park, MD), an inkjet printed SERS substrate; EnSpectr SERS

475 substrate (EnSpectr, Chernogolovka, Russia), a solid surface based substrate; and ATO ID ${ }^{\mathrm{TM}}$ Ag

476 and Au substrates (ATO ID, Vilnius, Lithuania), formed using ultrashort pulse laser on a soda-

477 lime glass substrate. Aside from the business aspect of selling their own patented SERS substrate,

478 these SERS substrates mostly utilize cheaper fabrication techniques to lower the cost of

479 production, thus reducing the sales price tag. However, the SERS substrates are still relatively

480 expensive, the cheapest running at around US\$18/substrate with a surface dimension of

$48112.5 \times 5 \mathrm{~mm}$. With such a small surface area per substrate, it might still not be feasible for high

482 throughput analysis in industrial applications. Thus, it is certainly an area that is growing, seeing

483 that the number of SERS publications focusing on substrate fabrication has continued to increase 484 rapidly.

One possible area of growth in this field is the use of controlled wet chemistry to

487 fabricate the SERS substrates, since these techniques do not often require expensive equipment

488 such as laser cutting. By optimizing the parameters used to control nanoparticle growth, these 
methods can potentially be used to create relatively cheap substrates. An example of this kind of

490 SERS substrates are the dendritic nanostructures made from metal reduction methods such as

491 from the reduction of $\mathrm{ZnO}$ or $\mathrm{Al}_{2} \mathrm{O}_{3}$ with $\mathrm{AgNO}_{3}$. The resultant substrate is a leaf like dendritic

492 nanostructure that are interlinked in even gaps with consistent spherical diameter (assuming

493 optimized conditions are used) [78,79]. Another possible commercial use of SERS substrates for

494 pesticides detection is filter-based substrates [80]. To fabricate this substrate, Ag NPs were

495 embedded onto a filter paper using a silver mirror reaction method. Then, in order to test

496 pesticide residues on fruits, the filter paper substrate was wiped onto the fruit surface. Limit of

497 detection of thiram and paraxoan was between $10^{-7}$ to $10^{-9} \mathrm{M}$ with less than $20 \% \mathrm{RSV}$, which is

498 considerably reliable for SERS measurements. As more studies are being conducted to

499 investigate the application of these cheaper alternative SERS substrates, the cost of

500 commercializing SERS substrates will potentially decrease drastically to allow for widespread

501 commercial applications.

502

In order to apply an analytical technique for practical and routine applications, it is

504 important get accepted and approved by respected organizations such as AOAC International.

505 Although there are thousands of different SERS substrates being fabricated and published, their

506 performance are often tested on different targets. Hence, it is difficult to compare the

507 performance between different substrates. Furthermore, the experimental methods and Raman

508 instrument/specifications such as laser power, aperture size and integration times used are

509 dependent on the researcher's choice since a standardized protocol is not available. Hence, there

510 are very few instructions for a research lab to conduct standard pesticide detection analysis using

511 SERS. This encourages them to look at more established methods such as GC/LC-MS. The 
512 discovery phase for making SERS substrates is becoming more mature, and hence a possible

513 future trend would be the implementation of good SERS substrates for standardized pesticide

514 detection analysis.

\section{3.2. Method integration and advance of instrumentation}

519 such as pesticides. However, like any analytical technique, there are limitations to the use of one

520 technique to perform detection assays. To overcome such limitations, integration with other

521 useful techniques can bring added advantages such as verification of results, better extraction

522 procedures, and automation of detection.

Such integration studies have become increasingly popular in recent years. In one study,

525 plasmonic nanoparticle modified capillary (NPMC) was fabricated and used for HPLC-SERS,

526 which not only separated the target pesticide, thiram, from its matrix but also gave molecular

527 specific fingerprints [81]. A similar separation procedure was integrated with SERS using thin

528 layer chromatography to detect methadathion [59]. To validate the results of Au NP based

529 colorimetric assays, SERS has been used since the nanoparticles when aggregated can form

530 localized surface plasmon resonance effect to enhance the Raman signals [82]. The

531 miniaturization of the SERS method in a confined space has also been investigated using the Ag

532 NP based microfluidic SERS assay to detect the pesticides alachlor and carbofuran [83] and the

533 pesticides methyl parathion and malachite green [84]. 
With more of these types of integration studies, the advantageous of SERS will be

536 reflected in many more studies and potentially be expanded for wider applications. Significant

537 advance in instrumentation is also critically important, in order to advance SERS as a routine and

538 cost- effective analytical technique.

\section{$540 \quad$ 3.3. Internalized pesticides analysis}

Another upcoming trend in the detection of pesticides using SERS is the ability to

542 analyze pesticides that have been internalized or penetrated into cells of living tissues. Based on

543 the physical behaviors of pesticides, they can be divided into two categories: systemic and non-

544 systemic. Systemic pesticides are able to penetrate into the plant tissues and translocate from the

545 site of applications to other parts of the plant. Non-systemic pesticides have little or no

546 penetration ability. In all the insitu SERS studies and many SERS studies that applied a simple

547 extraction procedure, their applications have been limited to pesticides on the surface. Therefore,

548 more studies are needed for detection of internalized pesticides.

In order to observe the internalization of trace levels of pesticides over time, SERS can be

551 conducted by adding penetrable nanoparticles that have strong adsorption affinity to the

552 pesticides of interest. Coupled with a confocal Raman microscope which can scan layer by layer

553 inside the plant tissue, it is able to study the penetration depth and location of the internalized

554 pesticides[85]. Our preliminary data showed that with increase incubation times, thiabendazole, a

555 systemic pesticide, can gradually penetrate further into a spinach leaf at $210 \mu \mathrm{m}$ depth after 2

556 days of exposure (Figure 4). 
558 By investigating the potential of using SERS for analyzing internalized pesticides, it 559 opens the door for not only the in situ detection of internal pesticides, but also for the

560 understanding of the mechanisms of pesticides behaviors and their fate. Understanding pesticide

561 penetration behaviors and fate will help us to develop a better strategy to safely and effectively

562 apply pesticides.

563

564 


\section{Conclusions}

566 SERS is a multi-versatile detection tool that has evolved to become a highly potential

567 rapid detection technique today. Its excellent sensitivity to the detection of a wide range of

568 pesticides has promoted its use as an alternative detection technique for rapid pesticide analysis.

569 The reproducibility, portability and detection in complex matrices have been improving as

570 evidenced by the increase in scientific articles addressing these topics. The expansion of SERS

571 as a tool for pesticides detection has also started to become a reality as the commercialization of

572 more cost effective substrates has become more common. Furthermore, expanded usage of SERS

573 through integration with other analytical techniques has broadened its usage scope. Lastly, new

574 areas of research such as the analysis of internalized pesticides in plants in situ are poised to

575 expand the popularity of SERS. Overall, the prospect of SERS as an alternative pesticide

576 detection tool seems feasible and should continue to attract more studies in this area of research.

577 Advancement in instrumentation and commercialization is expected to further apply SERS as a

578 routine and cost-effective analytical method for pesticide detection.

\section{Acknowledgments}

585 We acknowledge USDA-NIFA (award \# 2016-67017-24458) for supporting this work. 


\section{References}

[1] J. Pretty, Agricultural sustainability: concepts, principles and evidence., Philos. Trans. R. Soc. Lond. B. Biol. Sci. 363 (2008) 447-65. doi:10.1098/rstb.2007.2163.

[2] A. Grube, D. Donaldson, T. Kiely, L. Wu, Pesticides Industry Sales and Usage 2006 and 2007 Market Estimates, U.S. Environ. Prot. Agency. http://www (2011).

[3] F. Hernández, J. V Sancho, O.J. Pozo, Critical review of the application of liquid chromatography/mass spectrometry to the determination of pesticide residues in biological samples., Anal. Bioanal. Chem. 382 (2005) 934-46. doi:10.1007/s00216-005-3185-5.

[4] J.S. Aulakh, a. K. Malik, V. Kaur, P. Schmitt-Kopplin, A Review on Solid Phase Micro Extraction—High Performance Liquid Chromatography (SPME-HPLC) Analysis of Pesticides, Crit. Rev. Anal. Chem. 35 (2005) 71-85. doi:10.1080/10408340590947952.

[5] J. Sherma, Review of advances in the thin layer chromatography of pesticides: 20102012., J. Environ. Sci. Health. B. 48 (2013) 417-30. doi:10.1080/03601234.2012.761526.

[6] J. Sherma, Review of advances in the thin layer chromatography of pesticides: 20122014., J. Environ. Sci. Health. B. 50 (2015) 301-16. doi:10.1080/03601234.2015.1000163.

[7] D. Cialla, A. März, R. Böhme, F. Theil, K. Weber, M. Schmitt, et al., Surface-enhanced Raman spectroscopy (SERS): progress and trends., Anal. Bioanal. Chem. 403 (2012) 2754. doi:10.1007/s00216-011-5631-x.

[8] Y. Cao, J. Zhang, Y. Yang, Z. Huang, N.V. Long, C. Fu, Engineering of SERS Substrates Based on Noble Metal Nanomaterials for Chemical and Biomedical Applications, Appl. Spectrosc. Rev. 50 (2015) 499-525. doi:10.1080/05704928.2014.923901.

[9] C.L. Haynes, A.D. McFarland, R.P. Van Duyne, Surface-Enhanced Raman Spectroscopy, Anal. Chem. 77 (2005) 338 A-346 A. doi:10.1021/ac053456d. 
611 [10] J. Zheng, L. He, Surface-Enhanced Raman Spectroscopy for the Chemical Analysis of

612 Food, Compr. Rev. Food Sci. Food Saf. 13 (2014) 317-328. doi:10.1111/1541-

$613 \quad 4337.12062$.

614 [11] D.-W. Li, W.-L. Zhai, Y.-T. Li, Y.-T. Long, Recent progress in surface enhanced Raman spectroscopy for the detection of environmental pollutants, Microchim. Acta. 181 (2013)

[12] K.C. Bantz, A.F. Meyer, N.J. Wittenberg, H. Im, O. Kurtuluş, S.H. Lee, et al., Recent progress in SERS biosensing., Phys. Chem. Chem. Phys. 13 (2011) 11551-67.

[13] P.H.B. Aoki, L.N. Furini, P. Alessio, A.E. Aliaga, C.J.L. Constantino, Surface-enhanced Raman scattering (SERS) applied to cancer diagnosis and detection of pesticides, explosives, and drugs, Rev. Anal. Chem. 32 (2013). doi:10.1515/revac-2012-0019.

$$
\text { C. Muehlethaler, M. Leona, J.R. Lombardi, Review of Surface Enhanced Raman }
$$
Scattering Applications in Forensic Science, Anal. Chem. 88 (2016) 152-169. doi:10.1021/acs.analchem.5b04131.

626 [15] A.P. Craig, A.S. Franca, J. Irudayaraj, Surface-Enhanced Raman Spectroscopy Applied to Food Safety, (2013).

628 [16] L. Chen, J. Choo, Recent advances in surface-enhanced Raman scattering detection technology for microfluidic chips., Electrophoresis. 29 (2008) 1815-28. doi:10.1002/elps.200700554.

631 [17] B. Sharma, R.R. Frontiera, A.-I. Henry, E. Ringe, R.P. Van Duyne, SERS: Materials, applications, and the future, Mater. Today. 15 (2012) 16-25. doi:10.1016/S13697021(12)70017-2. 
634 [18] A.J. Haes, C.L. Haynes, A.D. McFarland, G.C. Schatz, R.P. Van Duyne, S. Zou,

635

636

637

638

639

640

641

642

643

644

645

646

647

648

649

650

651

652

653

654

655

656

Plasmonic Materials for Surface-Enhanced Sensing and Spectroscopy, MRS Bull. 30 (2005) 368-375. doi:10.1557/mrs2005.100.

[19] A.M. Alak, T. Vo-Dinh, Surface-enhanced Raman spectrometry of organo phosphorus chemical agents, Anal. Chem. 59 (1987) 2149-2153. doi:10.1021/ac00144a030.

[20] J. Zhao, A.O. Pinchuk, J.M. McMahon, S. Li, L.K. Ausman, A.L. Atkinson, et al., Methods for describing the electromagnetic properties of silver and gold nanoparticles., Acc. Chem. Res. 41 (2008) 1710-20. doi:10.1021/ar800028j.

[21] K. Kneipp, Y. Wang, H. Kneipp, L. Perelman, I. Itzkan, R. Dasari, et al., Single Molecule Detection Using Surface-Enhanced Raman Scattering (SERS), Phys. Rev. Lett. 78 (1997) 1667-1670. doi:10.1103/PhysRevLett.78.1667.

[22] H. Liu, L. Zhang, X. Lang, Y. Yamaguchi, H. Iwasaki, Y. Inouye, et al., Single molecule detection from a large-scale SERS-active Au79Ag21 substrate, Sci. Rep. 1 (2011) 11021106. doi:10.1038/srep00112.

[23] A.B. Zrimsek, N.L. Wong, R.P. Van Duyne, Single Molecule Surface-Enhanced Raman Spectroscopy: A Critical Analysis of the Bianalyte versus Isotopologue Proof, J. Phys. Chem. C. 120 (2016) 5133-5142. doi:10.1021/acs.jpcc.6b00606.

[24] E.C. Le Ru, P.G. Etchegoin, Single-Molecule Surface-Enhanced Raman Spectroscopy, Annu. Rev. Phys. Chem. 63 (2012) 65-87. doi:10.1146/annurev-physchem-032511143757.

[25] S.E.J. Bell, M.R. McCourt, SERS enhancement by aggregated Au colloids: effect of particle size., Phys. Chem. Chem. Phys. 11 (2009) 7455-62. doi:10.1039/b906049a.

[26] W.W. Yu, I.M. White, A simple filter-based approach to surface enhanced Raman 
spectroscopy for trace chemical detection, Analyst. 137 (2012) 1168. doi:10.1039/c2an15947c.

[27] Y.-T. Li, L.-L. Qu, D.-W. Li, Q.-X. Song, F. Fathi, Y.-T. Long, Rapid and sensitive insitu detection of polar antibiotics in water using a disposable Ag-graphene sensor based on electrophoretic preconcentration and surface-enhanced Raman spectroscopy., Biosens. Bioelectron. 43 (2013) 94-100. doi:10.1016/j.bios.2012.12.005.

D. Li, D.-W. Li, J.S. Fossey, Y.-T. Long, Portable surface-enhanced Raman scattering sensor for rapid detection of aniline and phenol derivatives by on-site electrostatic preconcentration., Anal. Chem. 82 (2010) 9299-305. doi:10.1021/ac101812x.

[29] B.D. J. Fang, H. You, P. Kong, Y. Yi, X. Song, Dendritic Silver Nanostructure Growth and Evolution in Replacement Reaction, Cryst. Growth Des. 7 (2007) 864-867. doi:10.1021/cg0604879. sonochemical synthesis of 3D nanotextured silver microflowers as highly efficient SERS substrates, J. Mater. Chem. 21 (2011) 18817. doi:10.1039/c1jm12831k. Spectrosc. 121 (2014) 63-9. doi:10.1016/j.saa.2013.10.013. (2014) 411-6. doi:10.1016/j.saa.2014.06.054.

[31] L. Zhang, B. Wang, G. Zhu, X. Zhou, Synthesis of silver nanowires as a SERS substrate for the detection of pesticide thiram., Spectrochim. Acta. A. Mol. Biomol. Spectrosc. 133

$$
\text { (2014) 411-6. doi:10.1016/j.saa.2014.06.054. }
$$

B. Wang, L. Zhang, X. Zhou, Synthesis of silver nanocubes as a SERS substrate for the determination of pesticide paraoxon and thiram., Spectrochim. Acta. A. Mol. Biomol. sensitive and reproducible surface-enhanced Raman scattering substrates, RSC Adv. 4 
(2014) 3487-3493. doi:10.1039/C3RA45935G.

681 [34] S. Lee, J. Choi, L. Chen, B. Park, J.B. Kyong, G.H. Seong, et al., Fast and sensitive trace analysis of malachite green using a surface-enhanced Raman microfluidic sensor, Anal. Chim. Acta. 590 (2007) 139-144. doi:10.1016/j.aca.2007.03.049.

[35] Y. Pan, X. Wang, H. Zhang, Y. Kang, T. Wu, Y. Du, Gold-nanoparticle, functionalizedporous-polymer monolith enclosed in capillary for on-column SERS detection, Anal.

[36] J. Zheng, S. Pang, T.P. Labuza, L. He, Semi-quantification of surface-enhanced Raman scattering using a handheld Raman spectrometer: a feasibility study., Analyst. 138 (2013) 7075-8. doi:10.1039/c3an01450a.

P. Guo, D. Sikdar, X. Huang, K.J. Si, W. Xiong, S. Gong, et al., Plasmonic core-shell nanoparticles for SERS detection of the pesticide thiram: size- and shape-dependent Raman enhancement., Nanoscale. 7 (2015) 2862-8. doi:10.1039/c4nr06429a.

[38] A. Kim, S.J. Barcelo, Z. Li, SERS-based pesticide detection by using nanofinger sensors., Nanotechnology. 26 (2015) 015502. doi:10.1088/0957-4484/26/1/015502.

T.H.D. Nguyen, Z. Zhang, A. Mustapha, H. Li, M. Lin, Use of graphene and gold nanorods as substrates for the detection of pesticides by surface enhanced Raman spectroscopy., J. Agric. Food Chem. 62 (2014) 10445-51. doi:10.1021/jf5036417. Electrophoretic-Ultraviolet Method for the Separation and Estimation of Zineb, Maneb, and Ferbam in Food Samples, (n.d.). method for the estimation of dithiocarbamate fungicide residues and safety evaluation of 
mancozeb in fruits and vegetables, Food Chem. 150 (2014) 175-181. doi:10.1016/j.foodchem.2013.10.148.

[42] O. Péron, E. Rinnert, M. Lehaitre, P. Crassous, C. Compère, Detection of polycyclic aromatic hydrocarbon (PAH) compounds in artificial sea-water using surface-enhanced Raman scattering (SERS), Talanta. 79 (2009) 199-204. doi:10.1016/j.talanta.2009.03.043.

[43] J. Kubackova, G. Fabriciova, P. Miskovsky, D. Jancura, S. Sanchez-Cortes, Sensitive surface-enhanced Raman spectroscopy (SERS) detection of organochlorine pesticides by alkyl dithiol-functionalized metal nanoparticles-induced plasmonic hot spots., Anal. Chem.

[44] H. Zheng, B. Zou, L. Chen, Y. Wang, X. Zhang, S. Zhou, Gel-assisted synthesis of oleate-modified Fe 304 @Ag composite microspheres as magnetic SERS probe for thiram detection, CrystEngComm. 17 (2015) 6393-6398. doi:10.1039/C5CE01017A.

W. Ren, Y. Fang, E. Wang, A Binary Functional Substrate for Enrichment and Ultrasensitive SERS Spectroscopic Detection of Folic Acid Using Graphene Oxide/Ag Nanoparticle Hybrids, ACS Nano. 5 (2011) 6425-6433. doi:10.1021/nn201606r.

F. Xia, X. Zuo, R. Yang, Y. Xiao, D. Kang, A. Vallée-Bélisle, et al., Colorimetric detection of DNA, small molecules, proteins, and ions using unmodified gold nanoparticles and conjugated polyelectrolytes., Proc. Natl. Acad. Sci. U. S. A. 107 (2010)

722 [47] J. Neng, M.H. Harpster, H. Zhang, J.O. Mecham, W.C. Wilson, P.A. Johnson, A versatile 723 SERS-based immunoassay for immunoglobulin detection using antigen-coated gold nanoparticles and malachite green-conjugated protein A/G., Biosens. Bioelectron. 26 (2010) 1009-15. doi:10.1016/j.bios.2010.08.015. 
726 [48] J.F. Li, Y.F. Huang, Y. Ding, Z.L. Yang, S.B. Li, X.S. Zhou, et al., Shell-isolated nanoparticle-enhanced Raman spectroscopy., Nature. 464 (2010) 392-5. doi:10.1038/nature08907.

[49] M. Keating, Y. Chen, I.A. Larmour, K. Faulds, D. Graham, Growth and surface-enhanced Raman scattering of Ag nanoparticle assembly in agarose gel, Meas. Sci. Technol. 23 (2012) 084006. doi:10.1088/0957-0233/23/8/084006.

732

733

734

735

736

737

738

739

740

[50] Y.-H. Lee, S. Dai, J.P. Young, Silver-doped sol-gel films as the substrate for surfaceenhanced Raman scattering, J. Raman Spectrosc. 28 (1997) 635-639. doi:10.1002/(SICI)1097-4555(199708)28:8<635::AID-JRS152>3.0.CO;2-0.

[51] D. Lee, S. Lee, G.H. Seong, J. Choo, E.K. Lee, D.-G. Gweon, et al., Quantitative Analysis of Methyl Parathion Pesticides in a Polydimethylsiloxane Microfluidic Channel Using Confocal Surface-Enhanced Raman Spectroscopy, Appl. Spectrosc. 60 (2006) 373377.

[52] S.-C. Luo, K. Sivashanmugan, J.-D. Liao, C.-K. Yao, H.-C. Peng, Nanofabricated SERSactive substrates for single-molecule to virus detection in vitro: A review, Biosens. Bioelectron. 61 (2014) 232-240. doi:10.1016/j.bios.2014.05.013.

[53] B. Sharma, M. Fernanda Cardinal, S.L. Kleinman, N.G. Greeneltch, R.R. Frontiera, M.G. Blaber, et al., High-performance SERS substrates: Advances and challenges, MRS Bull. 38 (2013) 615-624. doi:10.1557/mrs.2013.161.

[54] H. Fang, X. Zhang, S.J. Zhang, L. Liu, Y.M. Zhao, H.J. Xu, Ultrasensitive and quantitative detection of paraquat on fruits skins via surface-enhanced Raman spectroscopy, Sensors Actuators B Chem. 213 (2015) 452-456. doi:10.1016/j.snb.2015.02.121. 
[55] C.D.L. Albuquerque, R.J. Poppi, Detection of malathion in food peels by surfaceenhanced Raman imaging spectroscopy and multivariate curve resolution., Anal. Chim. Acta. 879 (2015) 24-33. doi:10.1016/j.aca.2015.04.019.

[56] B. Liu, P. Zhou, X. Liu, X. Sun, H. Li, M. Lin, Detection of Pesticides in Fruits by Surface-Enhanced Raman Spectroscopy Coupled with Gold Nanostructures, Food Bioprocess Technol. 6 (2012) 710-718. doi:10.1007/s11947-011-0774-5.

755

756

757

758

759

760

761

762

763

764

765

766

767

768

769

770

771

[57] R. Hou, S. Pang, L. He, In situ SERS detection of multi-class insecticides on plant surfaces, Anal. Methods. 7 (2015) 6325-6330. doi:10.1039/C5AY01058F.

[58] S. Pang, T.P. Labuza, L. He, Development of a single aptamer-based surface enhanced Raman scattering method for rapid detection of multiple pesticides., Analyst. (2014). doi:10.1039/c3an02263c.

[59] C. Yao, F. Cheng, C. Wang, Y. Wang, X. Guo, Z. Gong, et al., Separation, identification and fast determination of organophosphate pesticide methidathion in tea leaves by thin layer chromatography-surface-enhanced Raman scattering, Anal. Methods. 5 (2013) 5560. doi:10.1039/c3ay41152d.

[60] W.W. Yu, I.M. White, Inkjet-printed paper-based SERS dipsticks and swabs for trace chemical detection., Analyst. 138 (2013) 1020-5. doi:10.1039/c2an36116g.

[61] C.H. Lee, L. Tian, S. Singamaneni, Paper-based SERS swab for rapid trace detection on real-world surfaces., ACS Appl. Mater. Interfaces. 2 (2010) 3429-35. doi:10.1021/am1009875.

[62] L.-L. Qu, D.-W. Li, J.-Q. Xue, W.-L. Zhai, J.S. Fossey, Y.-T. Long, Batch fabrication of disposable screen printed SERS arrays., Lab Chip. 12 (2012) 876-81. doi:10.1039/c2lc20926h. 
772 [63] P.R. Stoddart, D.J. White, Optical fibre SERS sensors., Anal. Bioanal. Chem. 394 (2009)

773 1761-74. doi:10.1007/s00216-009-2797-6.

774 [64] F.L. Yap, P. Thoniyot, S. Krishnan, S. Krishnamoorthy, Nanoparticle Cluster Arrays for 775 High-Performance SERS through Directed Self-Assembly on Flat Substrates and on 776 Optical Fibers, ACS Nano. 6 (2012) 2056-2070. doi:10.1021/nn203661n.

777 [65] W. Wijaya, S. Pang, T.P. Labuza, L. He, Rapid detection of acetamiprid in foods using 778 779

780 surface-enhanced Raman spectroscopy (SERS)., J. Food Sci. 79 (2014) T743-7. doi:10.1111/1750-3841.12391.

[66] Z. Zhang, Q. Yu, H. Li, A. Mustapha, M. Lin, Standing gold nanorod arrays as reproducible SERS substrates for measurement of pesticides in apple juice and vegetables., J. Food Sci. 80 (2015) N450-8. doi:10.1111/1750-3841.12759.

[67] B.N. Khlebtsov, V.A. Khanadeev, E. V Panfilova, D.N. Bratashov, N.G. Khlebtsov, Gold nanoisland films as reproducible SERS substrates for highly sensitive detection of fungicides., ACS Appl. Mater. Interfaces. 7 (2015) 6518-29. doi:10.1021/acsami.5b01652.

[68] F. Barahona, C.L. Bardliving, A. Phifer, J.G. Bruno, C. a. Batt, An Aptasensor Based on Polymer-Gold Nanoparticle Composite Microspheres for the Detection of Malathion Using Surface-Enhanced Raman Spectroscopy, Ind. Biotechnol. 9 (2013) 42-50. doi:10.1089/ind.2012.0029.

[69] M. Procházka, Surface-Enhanced Raman Spectroscopy: Bioanalytical, Biomolecular and Medical Applications, Springer International Publishing, 2015. Raman spectroscopy substrates for in situ molecular sensing., ACS Appl. Mater.

794 Interfaces. 4 (2012) 6599-608. doi:10.1021/am302376q. 
795 [71] B. Liu, G. Han, Z. Zhang, R. Liu, C. Jiang, S. Wang, et al., Shell thickness-dependent 796 Raman enhancement for rapid identification and detection of pesticide residues at fruit peels., Anal. Chem. 84 (2012) 255-61. doi:10.1021/ac202452t.

798 [72] R.M. Stöckle, Y.D. Suh, V. Deckert, R. Zenobi, Nanoscale chemical analysis by tipenhanced Raman spectroscopy, Chem. Phys. Lett. 318 (2000) 131-136. doi:10.1016/S0009-2614(99)01451-7.

[73] X. Tang, W. Cai, L. Yang, J. Liu, Highly uniform and optical visualization of SERS substrate for pesticide analysis based on Au nanoparticles grafted on dendritic $\alpha$-Fe2O3., Nanoscale. 5 (2013) 11193-9. doi:10.1039/c3nr03671e.

[74] Y. Liu, B. He, Y. Zhang, H. Wang, B. Ye, Detection of Phosmet Residues on Navel

[75] Y. Fan, K. Lai, B.A. Rasco, Y. Huang, Analyses of phosmet residues in apples with Orange Skin by Surface-enhanced Raman Spectroscopy, Intell. Autom. Soft Comput. 21 surface-enhanced Raman spectroscopy, Food Control. 37 (2014) 153-157.

810 [76] Y. Liu, B. Ye, C. Wan, Y. Hao, A. Ouyang, Y. Lan, Determination of Dimethoate and Phosmet Pesticides with Surface-Enhanced Raman Spectroscopy, Spectrosc. Lett. (2014)

813 [77] Y. Fan, K. Lai, B.A. Rasco, Y. Huang, Determination of carbaryl pesticide in Fuji apples using surface-enhanced Raman spectroscopy coupled with multivariate analysis, LWT Food Sci. Technol. 60 (2015) 352-357. doi:10.1016/j.lwt.2014.08.011.

[78] L. He, M. Lin, H. Li, N.-J. Kim, Surface-enhanced Raman spectroscopy coupled with dendritic silver nanosubstrate for detection of restricted antibiotics, J. Raman Spectrosc. 
41 (2010) 739-744. doi:10.1002/jrs.2505.

819 [79] A. Gutés, C. Carraro, R. Maboudian, Silver dendrites from galvanic displacement on commercial aluminum foil as an effective SERS substrate., J. Am. Chem. Soc. 132 (2010) 1476-7. doi:10.1021/ja909806t.

822

[80] Y. Zhu, M. Li, D. Yu, L. Yang, A novel paper rag as "D-SERS” substrate for detection of pesticide residues at various peels., Talanta. 128 (2014) 117-24. doi:10.1016/j.talanta.2014.04.066.

[81] W. Wang, M. Xu, Q. Guo, Y. Yuan, R. Gu, J. Yao, Rapid separation and on-line detection by coupling high performance liquid chromatography with surface-enhanced Raman spectroscopy, RSC Adv. 5 (2015) 47640-47646. doi:10.1039/C5RA05562H.

T. Lang, S. Pang, L. He, Integration of colorimetric and SERS detection for rapid screening and validation of melamine in milk, Anal. Methods. 7 (2015) 6426-6431. doi:10.1039/C5AY00955C.

[83] J. Parisi, Q. Dong, Y. Lei, In situ microfluidic fabrication of SERS nanostructures for highly sensitive fingerprint microfluidic-SERS sensing, RSC Adv. 5 (2015) 14081-14089.

[84] S.H. Yazdi, I.M. White, Multiplexed detection of aquaculture fungicides using a pumpfree optofluidic SERS microsystem, in: E. Udd, G. Pickrell, H.H. Du, J.J. Benterou, X. Fan, A. Mendez, et al. (Eds.), SPIE Defense, Secur. Sens., International Society for Optics and Photonics, 2013: p. 87220V. doi:10.1117/12.2015528.

[85] T. Yang, Z. Zhang, B. Zhao, R. Hou, A. Kinchla, J.M. Clark, et al., Real-Time and in Situ Monitoring of Pesticide Penetration in Edible Leaves by Surface-Enhanced Raman Scattering Mapping, Anal. Chem. 88 (2016) 5243-5250. 
doi:10.1021/acs.analchem.6b00320.

842 [86] S.L. Clauson, J.M. Sylvia, T.A. Arcury, P. Summers, K.M. Spencer, Detection of Pesticides and Metabolites Using Surface-Enhanced Raman Spectroscopy (SERS): Acephate, Appl. Spectrosc. 69 (2015) 785-793.

[87] S. Huang, J. Hu, P. Guo, M. Liu, R. Wu, Rapid detection of chlorpyriphos residue in rice by surface-enhanced Raman scattering, Anal. Methods. 7 (2015) 4334-4339. doi:10.1039/C5AY00381D.

[88] Sun Xu-dong, Dong Xiao-ling, Quantitative Analysis of Dimethoate Pesticide Residues in Honey by Surface-Enhanced Raman Spectroscopy, Spectrosc. Spectr. Anal. 35 (2015)

[89] X. Wang, Y. Du, H. Zhang, Y. Xu, Y. Pan, T. Wu, et al., Fast enrichment and ultrasensitive in-situ detection of pesticide residues on oranges with surface-enhanced Raman spectroscopy based on Au nanoparticles decorated glycidyl methacrylate-ethylene dimethacrylate material, Food Control. 46 (2014) 108-114.

856 [90] Y. Zhang, Z. Wang, L. Wu, Y. Pei, P. Chen, Y. Cui, Rapid simultaneous detection of multi-pesticide residues on apple using SERS technique., Analyst. 139 (2014) 5148-54. doi:10.1039/c4an00771a.

859 [91] J.A. Webb, J. Aufrecht, C. Hungerford, R. Bardhan, Ultrasensitive analyte detection with Chem. C. 2 (2014) 10446-10454. doi:10.1039/C4TC01634C.

862 [92] L. He, T. Chen, T.P. Labuza, Recovery and quantitative detection of thiabendazole on 863 apples using a surface swab capture method followed by surface-enhanced Raman 
spectroscopy., Food Chem. 148 (2014) 42-6. doi:10.1016/j.foodchem.2013.10.023.

865 [93] X. Tang, R. Dong, L. Yang, J. Liu, Fabrication of Au nanorod-coated Fe 3 O 4 microspheres as SERS substrate for pesticide analysis by near-infrared excitation, J. Raman Spectrosc. 46 (2015) 470-475. doi:10.1002/jrs.4658.

868 [94] J.-K. Yang, H. Kang, H. Lee, A. Jo, S. Jeong, S.-J. Jeon, et al., Single-step and rapid 869 growth of silver nanoshells as SERS-active nanostructures for label-free detection of pesticides., ACS Appl. Mater. Interfaces. 6 (2014) 12541-9. doi:10.1021/am502435x.

871 [95] L. Zhang, C. Jiang, Z. Zhang, Graphene oxide embedded sandwich nanostructures for 872 enhanced Raman readout and their applications in pesticide monitoring., Nanoscale. 5 (2013) 3773-9. doi:10.1039/c3nr00631j.

874 [96] M. Fan, Z. Zhang, J. Hu, F. Cheng, C. Wang, C. Tang, et al., Ag decorated sandpaper as 875 flexible SERS substrate for direct swabbing sampling, Mater. Lett. 133 (2014) 57-59. doi:10.1016/j.matlet.2014.06.178. 
Figures/Tables

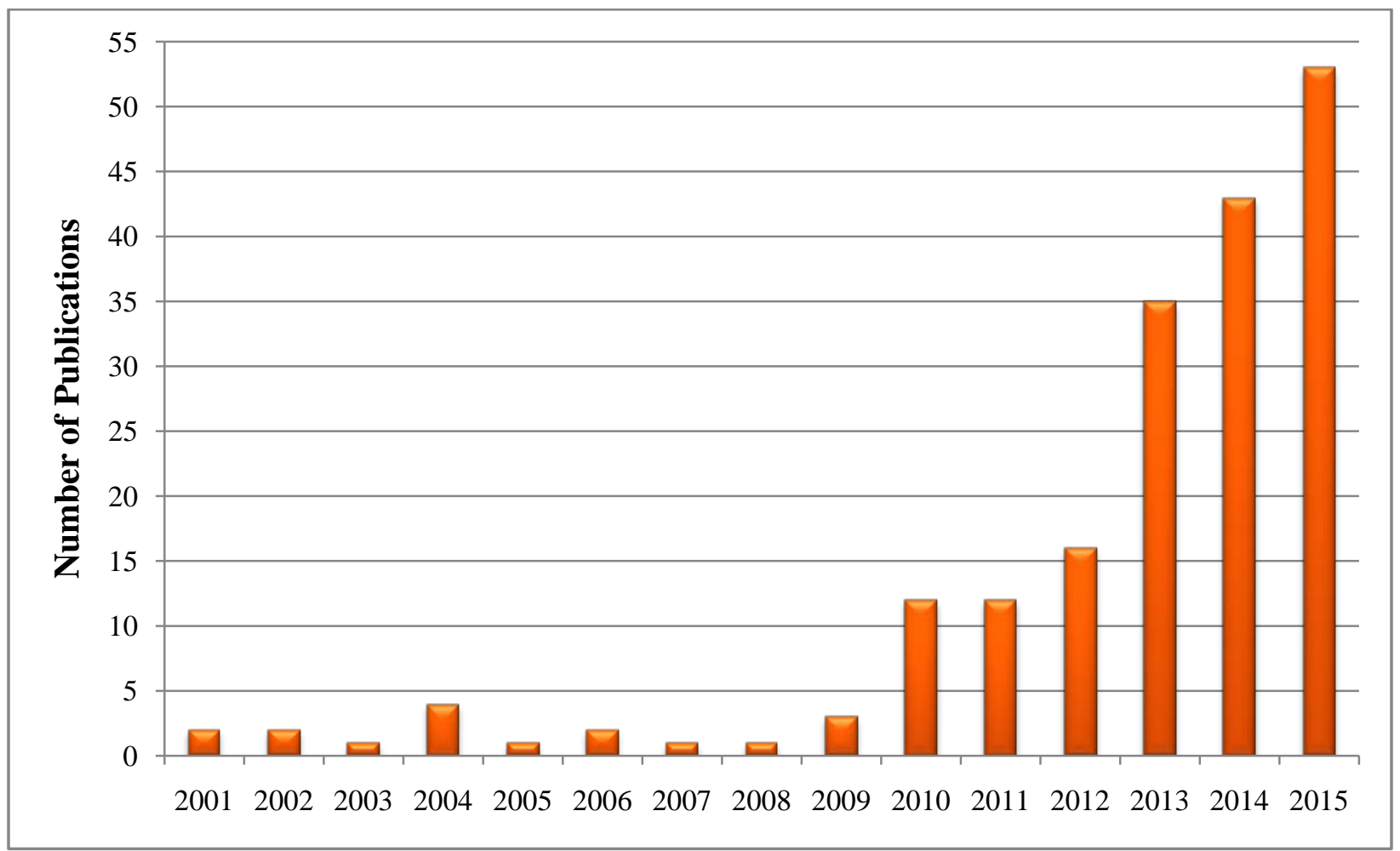

Fig. 1. Number of publications related to SERS detection of pesticides every year. Web of

882 Science ${ }^{\mathrm{TM}}$ was used with the following search terms: "Pesticides" and "SERS". Date Accessed:

$8831 / 15 / 2015$.

884 


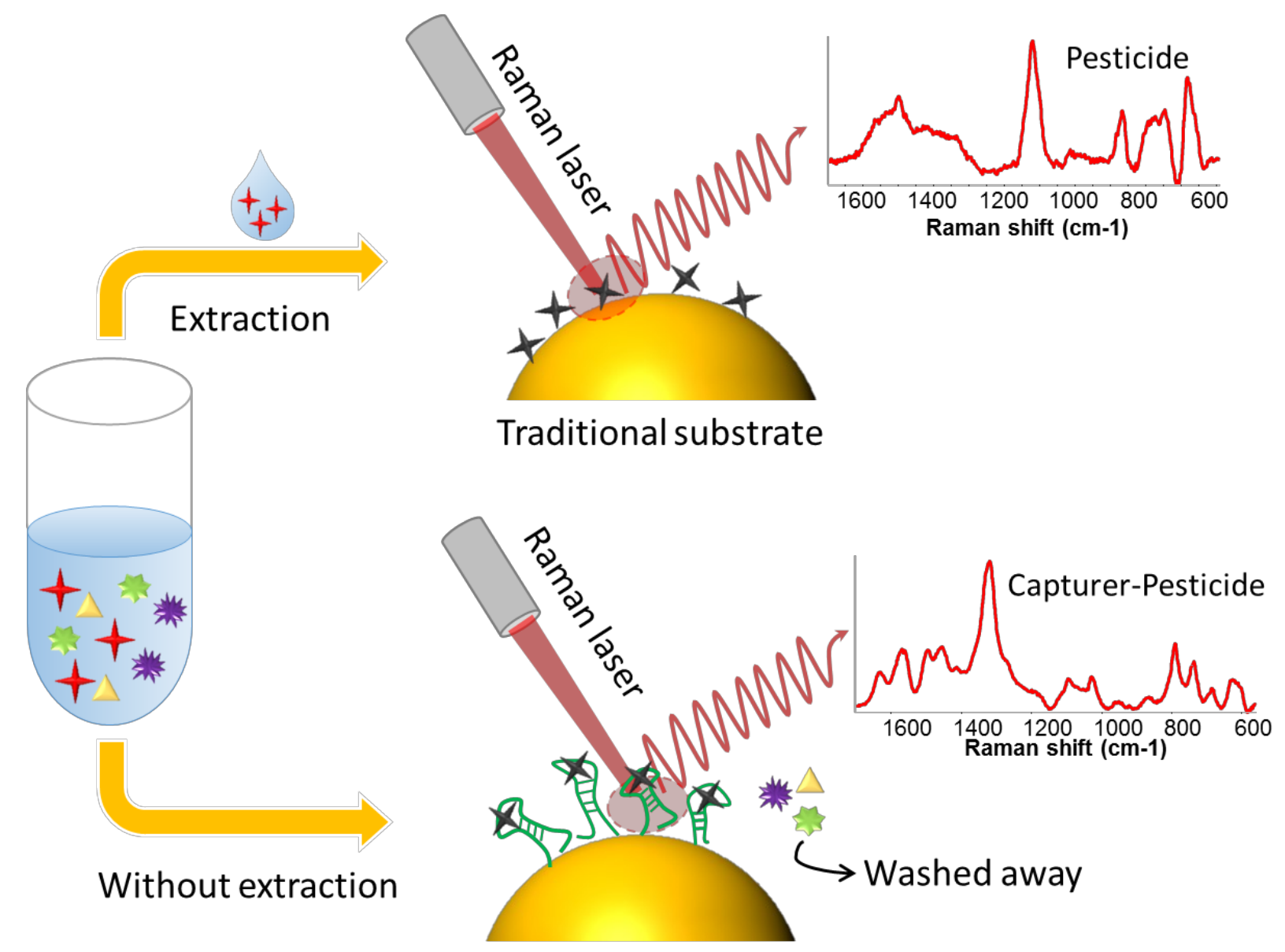

Functionalized substrate

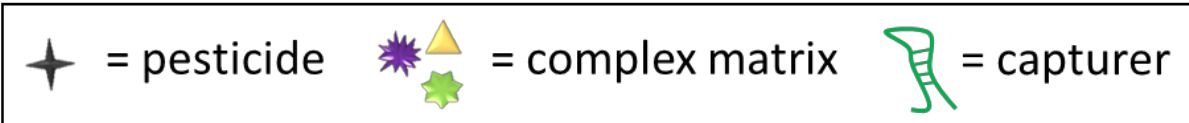

887

888 Fig. 2. Illustration of the detection of pesticides in real, complex matrix using extraction 889 procedure and substrate functionalization respectively. 
891

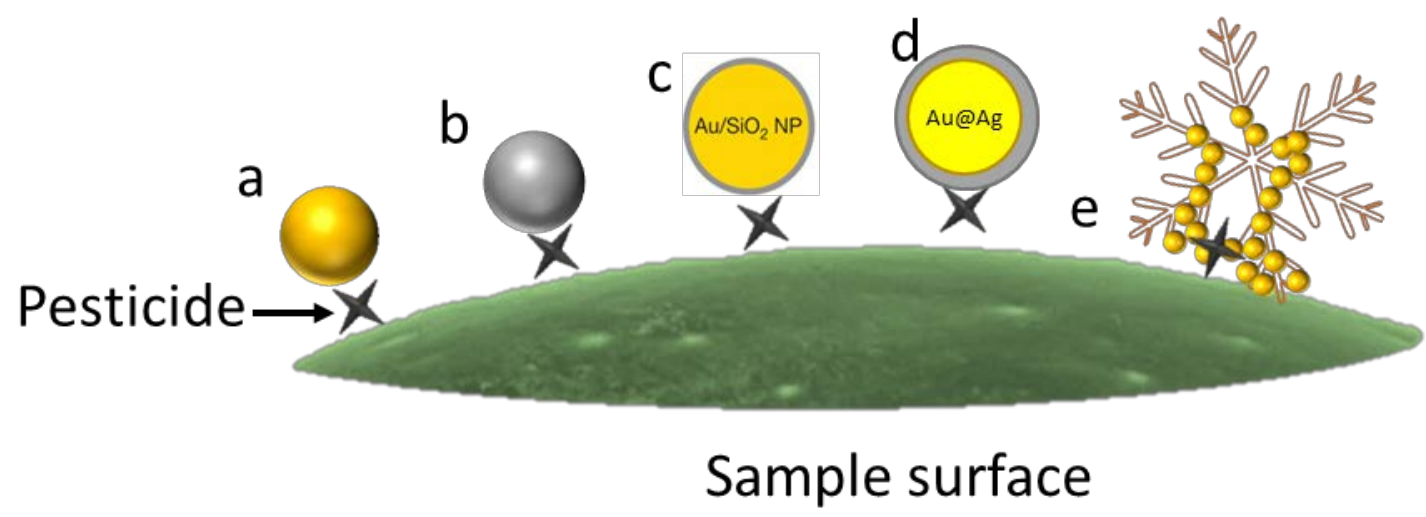

892

893

894

895 Fig. 3. SERS substrates applied for in situ pesticide detection. a. AuNPs [57] and [60], b. Ag

896 NPs (fabricated in situ) [70], c. Au@SiO NPs [48], d. Au@Ag NPs [71], e. Au NPs grafted on 897 dendritic $\alpha$-Fe2O3 [73].

898 
899
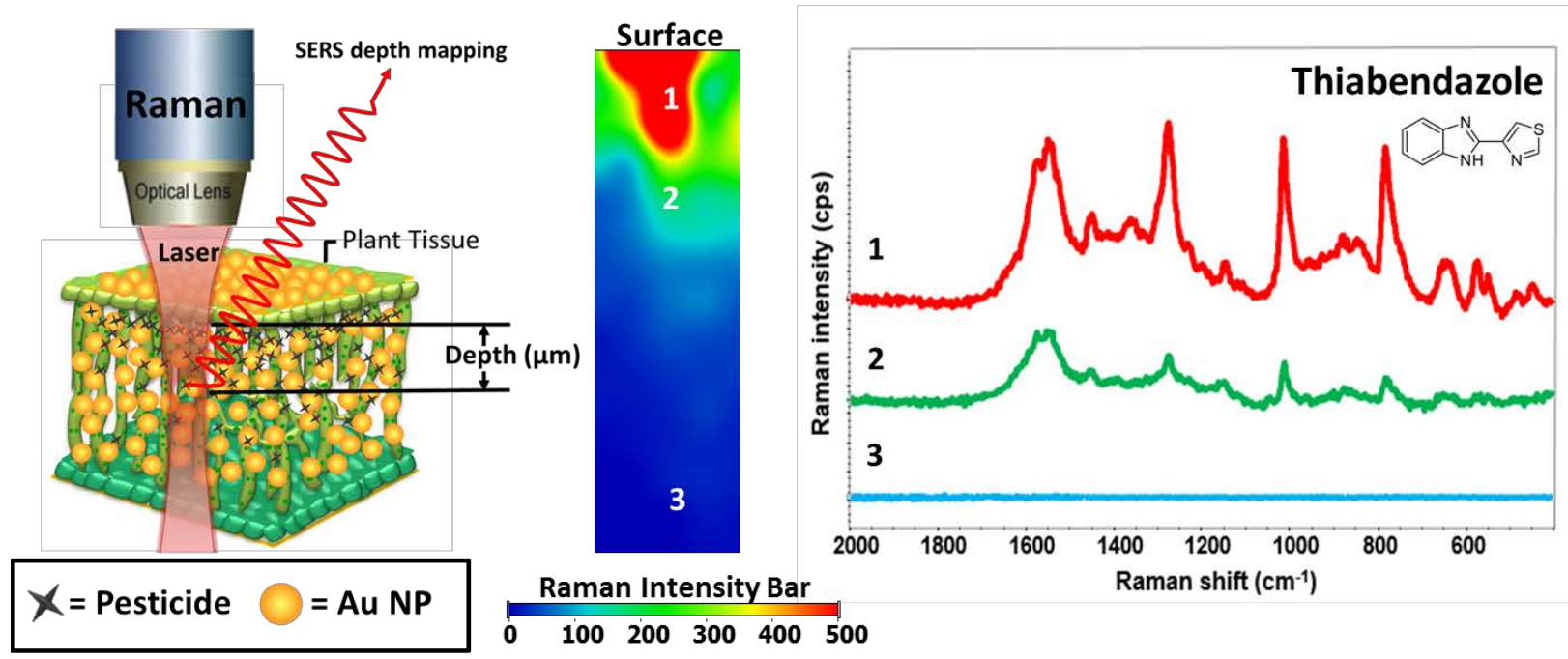

901

902 Fig. 4. Internalized pesticide analysis. Sample is immersed in Au NPs solution, and then SERS

903 depth analysis is performed. Right below the surface is where the majority of pesticides were

904 found (indicated in red). As the SERS spectra was taken deeper into the sample, the intensity of

905 the peaks decreased.

906

907 
Table 1

909 Summary of pesticides, detection matrices, nanosubstrates used, and the limit of detection (LOD).

910

\begin{tabular}{|c|c|c|c|c|c|}
\hline Pesticide & Class & Matrix & Substrate & LOD & Ref \\
\hline acephate & organophosphate & Urine & Au/Ag @Ti roughened & $19 \mathrm{ppb}$ & {$[86]$} \\
\hline azinphos-methyl & organophosphate & Apples / Tomatoes & AuNPs & $6.66 \mathrm{ppm} / 2.94 \mathrm{ppm}$ & [56] \\
\hline carbaryl & carbamate & $\begin{array}{l}\text { Apple juice / } \\
\text { Cabbage }\end{array}$ & Au-nanorod on silicon slide & $2.5 \mathrm{ppm} / 2.5 \mathrm{ppm}$ & [66] \\
\hline carbaryl & carbamate & Apples / Tomatoes & AuNPs & $4.51 \mathrm{ppm} / 5.35 \mathrm{ppm}$ & [56] \\
\hline chlorpyrifos & organophosphate & Water / Apple skin & Au nanofinger chips & $35 \mathrm{ppt}$ & [38] \\
\hline chlorpyriphos & organophosphate & Rice & OTR202 and 203 solution & $0.5 \mathrm{ppm}$ & [87] \\
\hline deltamethrin & pyrethroid & $\begin{array}{l}\text { Tea leaf / Apple } \\
\text { peel }\end{array}$ & AuNPs & $0.5 \mathrm{ppm} / 0.02 \mathrm{ppm}$ & [57] \\
\hline dimethoate & organophosphate & Honey & Klarite $^{\mathrm{TM}}$ & $2 \mathrm{ppm}$ & [88] \\
\hline disulfoton & organophosphate & Orange & AuNPs decorated GMA-EDMA & $1 \mathrm{ppb}, 39.7 \mathrm{mg} / \mathrm{kg}$ & [89] \\
\hline imidacloprid & neonicotinoid & $\begin{array}{l}\text { Tea leaf / Apple } \\
\text { peel }\end{array}$ & AuNPs & $0.5 \mathrm{ppm} / 0.02 \mathrm{ppm}$ & [57] \\
\hline isocarbophos & organophosphate & $\begin{array}{l}\text { Tea leaf / Apple } \\
\text { peel }\end{array}$ & AuNPs & $0.25 \mathrm{ppm} / 0.01 \mathrm{ppm}$ & [57] \\
\hline malathion & organophosphate & Food peels & AuNPs & $0.123 \mathrm{mg} \mathrm{L}^{-1}$ & [55] \\
\hline methamidophos & organophosphate & Apple & Au@Ag NRs & $440 \mu \mathrm{M}$ & [90] \\
\hline methyl parathion & organophosphate & apple & $\begin{array}{l}\text { Filter paper withmultibranched Au } \\
\text { nanoantennas (MGNs) }\end{array}$ & $26.3 \mu \mathrm{g}$ & [91] \\
\hline paraquat & viologen & Fruit skins & AgNPs & $1 \mathrm{nM}$ & [54] \\
\hline phorate & organophosphate & $\begin{array}{l}\text { Tea leaf / Apple } \\
\text { peel }\end{array}$ & AuNPs & $0.25 \mathrm{ppm} / 0.01 \mathrm{ppm}$ & [57] \\
\hline phosmet & organophosphate & Apples / Tomatoes & AuNPs & $6.51 \mathrm{ppm} / 2.91 \mathrm{ppm}$ & [56] \\
\hline phosmet & organophosphate & Apple extract & Klarite $^{\mathrm{TM}}$ & $1 \mathrm{ppm}$ & [75] \\
\hline phosmet & organophosphate & Orange & AuNPs decorated GMA-EDMA & $5 \mathrm{ppb}, 8.25 \mathrm{mg} / \mathrm{kg}$ & [89] \\
\hline thiabendazole & benzimidazole & Water / Apple skin & Au nanofinger chips & $1 \mathrm{ppb} / 7 \mathrm{ppb}$ & [38] \\
\hline thiabendazole & benzimidazole & Apple & Ag dendrites & $0.1 \mathrm{ppm}$ & [92] \\
\hline thiram & dithiocarbamate & Apple skin & Au nanoisland film & $5 \mathrm{ppb}$ & [67] \\
\hline thiram & dithiocarbamate & Orange & $\begin{array}{l}\text { plasmonic nanoparticle-modified capillary } \\
\text { (NPMC) }\end{array}$ & $100 \mathrm{nM}$ & [81] \\
\hline thiram & dithiocarbamate & Tea leaf & optofluidic SERS & $5 \mathrm{ppb}$ & [84] \\
\hline thiram & dithiocarbamate & Apple skin & Au nanorod-coated Fe3O4 microspheres & $100 \mathrm{nM}$ & [93] \\
\hline thiram & dithiocarbamate & Apple & Au@Ag NRs & $460 \mathrm{nM}$ & [90] \\
\hline thiram & dithiocarbamate & Apple skin & $\begin{array}{l}\text { Ag nanoshells (Ag NSs) around silica } \\
\text { NPs }\end{array}$ & $38 \mathrm{ng} \mathrm{cm}^{-2}$ & [94] \\
\hline thiram & dithiocarbamate & Grape & $\begin{array}{l}\text { Au@Ag NPs/GO/Au@Ag NPs sandwich } \\
\text { nanostructure }\end{array}$ & $0.03 \mathrm{ppm}$ & [95] \\
\hline triazophos & organophosphate & $\begin{array}{l}\text { Pear / } \\
\text { Tree leaf / } \\
\text { Plastic / } \\
\text { Glass }\end{array}$ & Ag coated Sandpaper & $\begin{array}{l}\sim 0.3 \mathrm{~cm}^{2}, 53.3 \mathrm{pM} \mathrm{cm}^{-2} / \\
\sim 0.6 \mathrm{~cm}^{2}, 266 \mathrm{pM} \mathrm{cm}^{-2} / \\
\sim 3 \mathrm{~cm}^{2}, 10.5 \mathrm{pM} \mathrm{cm}^{-2} / \\
\sim 4 \mathrm{~cm}^{2}, 4.2 \mathrm{pM} \mathrm{cm}^{-2}\end{array}$ & [96] \\
\hline
\end{tabular}

911 